\title{
Systematic drug sensitivity testing reveals synergistic growth inhibition by dasatinib or mTOR inhibitors with paclitaxel in ovarian granulosa cell tumor cells
}

\author{
Haltia, Ulla-Maija
}

2017-03

Haltia , U-M , Andersson, N , Yadav , B , Farkkila , A, Kulesskiy , E , Kankainen , M , Tang , J, Butzow, R, Riska, A , Leminen , A , Heikinheimo , M , Kallioniemi , O , Unkila-Kallio , L , Wennerberg , K, Aittokallio , T \& Anttonen, M 2017 , ' Systematic drug sensitivity testing reveals synergistic growth inhibition by dasatinib or mTOR inhibitors with paclitaxel in ovarian granulosa cell tumor cells ' , Gynecologic Oncology , vol. 144 , no. 3 , pp. 621-630 . https://doi.org/10.1016/j.

http://hdl.handle.net/10138/234618

https://doi.org/10.1016/j.ygyno.2016.12.016

publishedVersion

Downloaded from Helda, University of Helsinki institutional repository.

This is an electronic reprint of the original article.

This reprint may differ from the original in pagination and typographic detail.

Please cite the original version. 


\title{
Systematic drug sensitivity testing reveals synergistic growth inhibition by dasatinib or mTOR inhibitors with paclitaxel in ovarian granulosa cell tumor cells
}

\author{
Ulla-Maija Haltia a,b,1, Noora Andersson $^{\mathrm{a}, 1}$, Bhagwan Yadav ${ }^{\mathrm{c}, 1}$, Anniina Färkkilä a,b,*, Evgeny Kulesskiy ${ }^{\mathrm{c}}$, \\ Matti Kankainen ${ }^{\text {c }}$, Jing Tang ${ }^{\text {c }}$, Ralf Bützow ${ }^{\mathrm{d}}$, Annika Riska ${ }^{\mathrm{b}}$, Arto Leminen ${ }^{\mathrm{b}}$, Markku Heikinheimo ${ }^{\mathrm{a}, \mathrm{e}}$, \\ Olli Kallioniemi ${ }^{c}$, Leila Unkila-Kallio ${ }^{\mathrm{b}}$, Krister Wennerberg ${ }^{\mathrm{c}}$, Tero Aittokallio ${ }^{\mathrm{c}, \mathrm{g}}$, Mikko Anttonen ${ }^{\mathrm{f}}$ \\ a Children's Hospital, University of Helsinki and Helsinki University Hospital, PO Box 20, 00014 University of Helsinki, Finland \\ b Obstetrics and Gynecology, University of Helsinki and Helsinki University Hospital, PO Box 140, 00290 Helsinki, Finland \\ ' Institute for Molecular Medicine Finland (FIMM), University of Helsinki, PO Box 20, 00014 Helsinki, Finland \\ d Pathology, University of Helsinki and HUSLAB, Helsinki University Hospital, PO Box 400, 00290 Helsinki, Finland \\ e Department of Pediatrics, Washington University School of Medicine, St Louis Children's Hospital, St Louis, MO 63110, USA \\ f Clinical Chemistry and Hematology, University of Helsinki and HUSLAB, Helsinki University Hospital, PO Box 400, 00290 Helsinki, Finland \\ ${ }^{g}$ Department of Mathematics and Statistics, University of Turku, 20014 Turku, Finland
}

\section{H I G H L I G H T S}

- Systematic drug and molecular profiling discovers new therapies in ovarian cancer.

- AGCT cells show selective sensitivity to a TKI dasatinib and mTOR inhibitors.

- Dasatinib or mTOR inhibitors synergize with paclitaxel in AGCT cells.
G R A P H I C A L A B S T R A C T

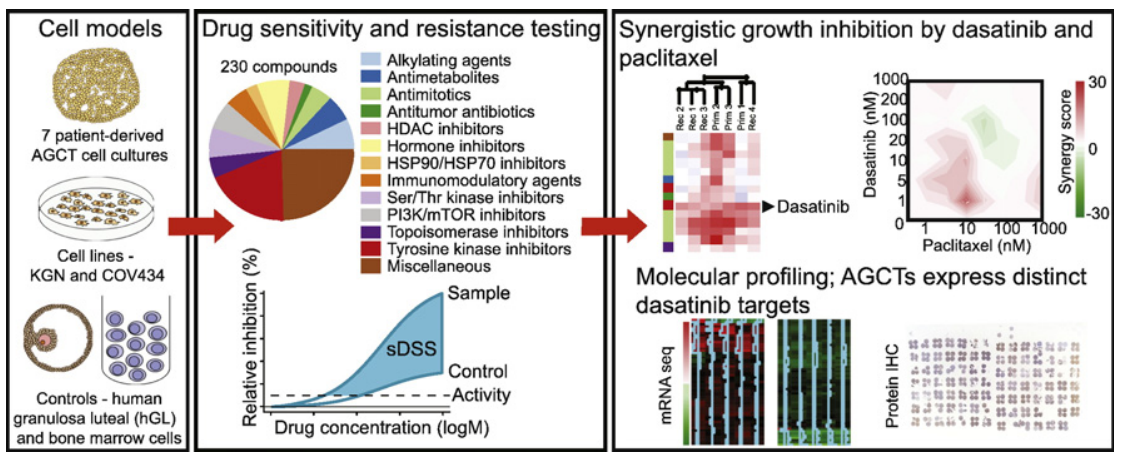

\section{A R T I C L E I N F O}

\section{Article history:}

Received 26 October 2016

Received in revised form 18 December 2016

Accepted 19 December 2016

Available online 16 January 2017

\section{Keywords:}

Granulosa cell tumor

Kinase inhibitors

Ovarian cancer

Screening strategies

\begin{abstract}
A B S T R A C T
Objective. Resistance to standard chemotherapy poses a major clinical problem in the treatment of ovarian cancer patients. Adult-type granulosa cell tumor (AGCT) is a unique ovarian cancer subtype for which efficient treatment options are lacking in advanced disease. To this end, systematic drug response and transcriptomics profiling were performed to uncover new therapy options for AGCTs.

Methods. The responses of three primary and four recurrent AGCTs to 230 anticancer compounds were screened in vitro using a systematic drug sensitivity and resistance testing (DSRT) platform, coupled with mRNA sequencing. The responses of the AGCTs were compared with those of human granulosa luteal cells and bone marrow mononuclear cells.

Results. Patient-derived AGCT cells showed selective sensitivity to the Src family tyrosine kinase inhibitor dasatinib. A combination of either dasatinib or an mTOR-inhibitor everolimus with paclitaxel resulted in synergistic inhibition of AGCT cell viability. The key kinase targets of dasatinib and members of the mTOR pathway
\end{abstract}

\footnotetext{
* Corresponding author at: Department of Obstetrics and Gynecology, Helsinki University Hospital, PO Box 400, 00290 Helsinki, Finland E-mail address: anniina.farkkila@helsinki.fi (A. Färkkilä).

${ }^{1}$ Equal contribution to this study.
} 
were constantly expressed at mRNA and protein levels, indicating multikinase signal addictions in the AGCT cells. Transcriptomic characterization of the tumors revealed no known oncogenic mutations, suggesting that the drug sensitivity of AGCTs was rather conveyed by selective target expression.

Conclusions. We used a systematic functional approach to reveal novel treatment options for a unique gynecological cancer. The selective synergy found between taxanes and dasatinib or mTOR inhibitors warrants further clinical investigations of these combinations in relapsed or aggressive AGCTs and demonstrate that highthroughput drug screening and molecular profiling can provide an effective approach to uncover new therapy options.

(C) 2016 Elsevier Inc. All rights reserved.

\section{Introduction}

Adult-type granulosa cell tumor (AGCT) is a unique sex-cord derived ovarian malignancy [1], characterized by a single nucleotide missense mutation in transcription factor FOXL2 (c.402C > G; C134W) [2]. The majority of AGCTs are diagnosed at an early stage, with an indolent clinical course [3]. However, up to $30 \%$ of the tumors recur [4,5], and $50-70 \%$ of relapsed patients will eventually die of disease-related complications [5, 6]. Radical surgery is the primary treatment of AGCT, but additional chemotherapy is needed in advanced stages and recurrent disease. Since the 1980s, AGCT has been treated mainly with platinum-based (cisplatin or carboplatin) chemotherapeutics, mostly in combination with either bleomycin and etoposide (BEP/BEC) or taxanes (principally paclitaxel). The response rates to BEP treatment vary from 37 to $83 \%$ in older studies $[7,8]$, whereas more recent evaluation of a small cohort of patients who were classified using RECIST criteria reported that the response rate to BEP was only $22 \%[9,10]$. The efficacy of paclitaxel in AGCT treatment is similar to that of BEP [8]. Thus, new treatments for advanced AGCTs are urgently needed. The relative rarity of the tumor type makes randomized clinical trials challenging and underlines the need for preclinical approaches.

AGCTs are considered chromosomally stable, and apart from the mutation in FOXL2, frequent mutations in common oncogenes or tumor suppressor genes have not been identified [1]. The pathogenesis of AGCTs is thought to be driven by transcriptional changes caused by the mutated FOXL2, leading to misregulation of proliferation and apoptosis $[11,12]$. There are, however, no therapeutic means to specifically target FOXL2 402C->G mutation-mediated alterations in AGCT.

In the present study, we used an established high-throughput drug sensitivity and resistance testing (DSRT) [13-15], in combination with molecular and genomic profiling, with the aim to uncover new therapeutic options for AGCTs. We identified potential AGCT-selective compounds, verified the expression of their target proteins, and performed RNA sequencing with variant analysis in the same AGCT samples. A combination of targeted agents, based on either dasatinib or mTOR inhibitors combined with paclitaxel, resulted in synergistic inhibition of AGCT cell viability. As these targeted drugs are approved for treating other malignancies, these results point to a straightforward drug repurposing strategy for the treatment of inoperable or chemoresistant AGCTs.

\section{Materials and methods}

\subsection{Primary AGCT cultures and GCT cell lines}

The ethics committee of Helsinki University Central Hospital and the National Supervisory Authority for Welfare and Health in Finland approved this study. Informed consent was obtained from the patients $(N=7)$ who donated fresh AGCT tissue for the study. The clinical characteristics of the AGCT patients are presented in Table 1. All AGCTs were positive for the FOXL2 402C $>\mathrm{G}$ mutation (Supplementary methods). Short-term primary tumor cell cultures were established as described [16] from three primary and four recurrent AGCTs during 2012-2015. All primary cultured AGCT samples expressed known AGCT markers (ER $\beta$, FOXL2, GATA4, AMHRII and inhibin- $\alpha$ ) (data not shown). The primary AGCT cells were cultured for 4-7 days to increase the cell number in Dulbecco's modified Eagle's medium (DMEM)/Ham's F-12 medium, supplemented with $10 \% \mathrm{FBS}$, penicillin/streptomycin, and L-glutamine (Gibco, Grand Island, NY, USA) in a humidified environment at $37{ }^{\circ} \mathrm{C}$ and $5 \% \mathrm{CO}_{2}$. The cells were trypsinized, counted, and suspended in the above-mentioned medium for high-throughput DSRT.

The KGN cell line [17] originating from a recurrent AGCT was confirmed to harbor the FOXL2 c.402C $>$ G mutation and was used for both DSRT and drug combination experiments. The COV434 cell line [18], which originates from a juvenile GCT, was also used for DSRT and drug combination experiments. COV434 does hot harbor the FOXL2 mutation and lacks also FOXL2 protein expression. Both cell lines were obtained from the Riken BioResource Center. The KGN and COV434 cells

Table 1

Patient characteristics.

\begin{tabular}{|c|c|c|c|c|c|c|c|}
\hline Features & Prim1 & Prim2 & Prim3 & Rec1 & $\operatorname{Rec} 2$ & $\operatorname{Rec} 3$ & $\operatorname{Rec} 4$ \\
\hline Tumor type & Primary & Primary & Primary & $\begin{array}{l}\text { Recurrence } \\
\text { n:o I }\end{array}$ & $\begin{array}{l}\text { Recurrence } \\
\text { n:o I }\end{array}$ & $\begin{array}{l}\text { Recurrence } \\
\text { n:o V }\end{array}$ & $\begin{array}{l}\text { Recurrence } \\
\text { n:o I }\end{array}$ \\
\hline Primary tumor stage & Ia & Ia & Ia & Ic & Ia & Ic & Ic \\
\hline Age at tumor in study/years from diagnosis & $62 / 0$ & $61 / 0$ & $56 / 0$ & $66 / 27$ & $82 / 9$ & $60 / 25$ & $66 / 12$ \\
\hline Tumor site & Right ovary & Right ovary & Left ovary & Pelvis & Pelvis & Pelvis & Pelvis \\
\hline Tumor size $(\mathrm{cm})^{\mathrm{a}}$ & 21 & 5 & 5 & 7 (3 tumors) & 16 (2 tumors) & 25 ( 4 tumors) & 17 \\
\hline Tumor subtype & Diffuse & Diffuse & Trabecular & Diffuse & Trabecular & Diffuse & Trabecular \\
\hline Prior adjuvant therapies & No & No & No & No & No & Yes $^{\mathrm{b}}$ & No \\
\hline Treatment of tumor in study & Surgery only & Surgery only & Surgery only & Surgery and BEC $\times 4$ & Surgery only & Surgery only & Palliative treatment \\
\hline New recurrence/follow-up (months) & $\mathrm{No} / 37$ & $\mathrm{No} / 28$ & No/15 & Yes $/ 12$ & Yes $/ 3$ & Yes/15 & - \\
\hline
\end{tabular}

Abbreviations BEC: bleomycin, etoposide, carboplatin.

a Tumor size measured as the longest diameter measured upon operation, and if multiple tumors, the diameters were summed.

b BEC 13 cycles in 2004-2005 and 2007 in total, epirubicin + carboplatin 6 cycles in 2009, letrozole 2,5 mg $\times 1$ from 2011 . 
were cultured in DMEM:F12 and DMEM, respectively, supplemented with $10 \%$ FBS, penicillin/streptomycin, and L-glutamine (Gibco), and passaged for $<6$ months following receipt or resuscitation of a frozen cell vial that tested negative for mycoplasma infection.

\subsection{Control cell models}

As controls, three samples of pooled human granulosa-luteal (hGL) cells obtained from women undergoing in vitro fertilization treatment at the Department of Obstetrics and Gynecology, Helsinki University Hospital were screened. hGL cells are mural benign granulosa cells from hCG-primed preovulatory follicles. Each of the three hGL pools consisted of granulosa-luteal cells derived from 78 to 122 mature ovulatory follicles from four to seven different patients. The hGL cells were isolated as previously described [19], and identified wild-type for the FOXL2 402C $>$ G mutation. Prior to the DSRT screen, freshly isolated hGL cells were suspended in DMEM/F12 growth medium, supplemented with $2.5 \%$ Nu-serum I, ITS $+{ }^{\text {TM }}$ Premix (both from BD Biosciences,
Bedford, MA, USA), penicillin/streptomycin, and L-glutamine (Gibco). As a second control cell model, DSRT data derived from bone marrow mononuclear cells from seven healthy donors were used [14]. To identify AGCT-selective responses, AGCT cell responses were compared to those from a collection of 68 cell lines derived from other solid tumors, including breast [20], ovarian, lung, and pancreatic cancers.

\subsection{DSRT}

DSRT was performed as previously described [14] on primary cultured AGCT, hGL, bone marrow mononuclear, KGN, and COV434 cells using a panel of 230 compounds (Supplementary Table S1). Twenty microliters of a single cell suspension (1000 cells/well for the primary AGCT cells and KGN and COV434 cell lines and 1000-2000 cells/well for the hGL cells) were transferred to each predrugged well using a MultiDrop Combi (Thermo Scientific, Waltham, MA, USA) peristaltic dispenser. After $72 \mathrm{~h}$ of incubation, the cell viability was measured
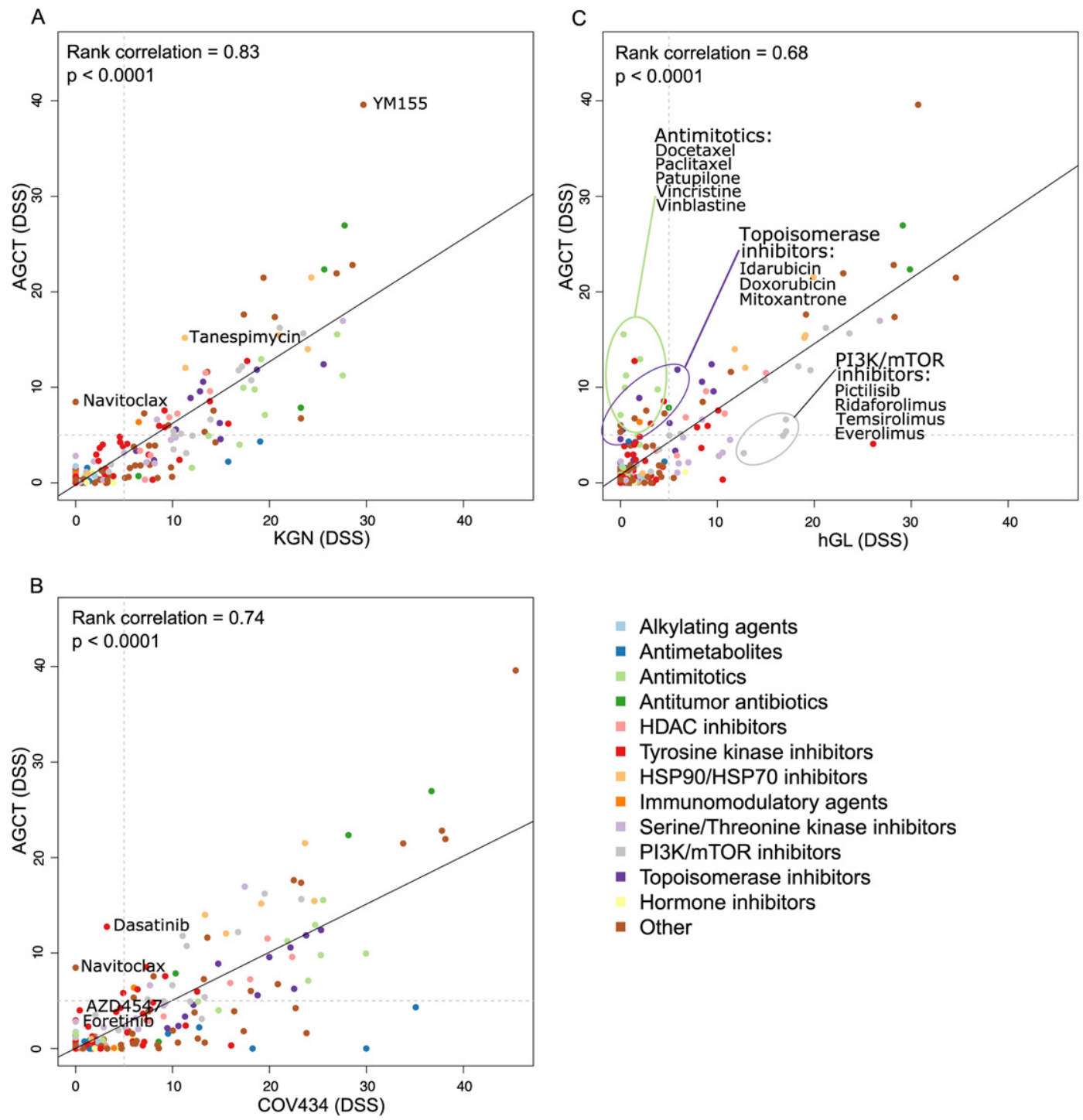

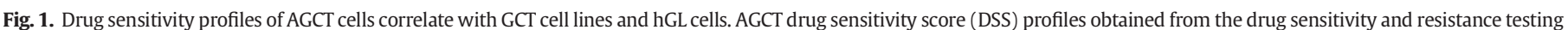

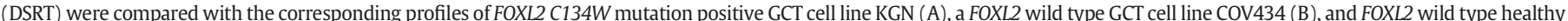

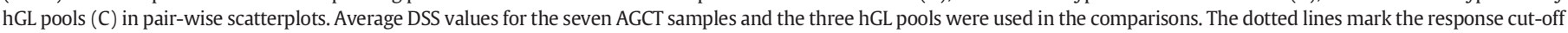

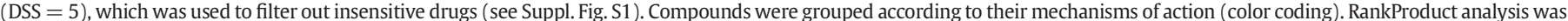

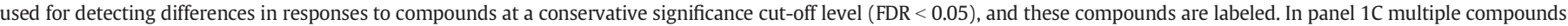

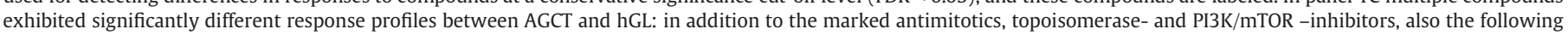
compounds showed differential responses: dasatinib, (+)JQ1, PF477736, obatoclax, linsitinib, SNS-032, alvocidib, BMS-754807 and YM155. 


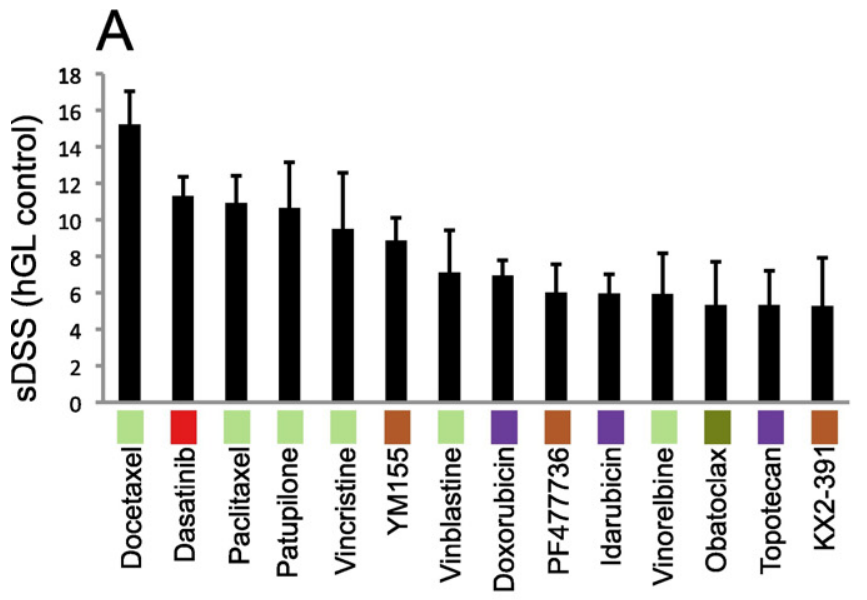

B sDSS (hGL control)

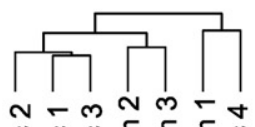

О워을 틀

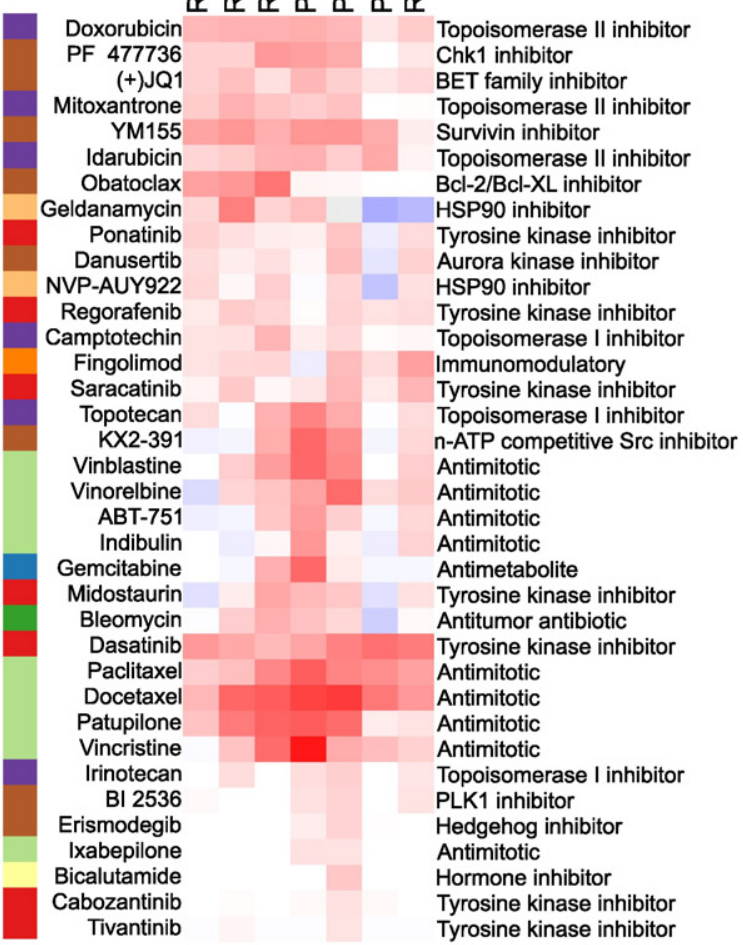

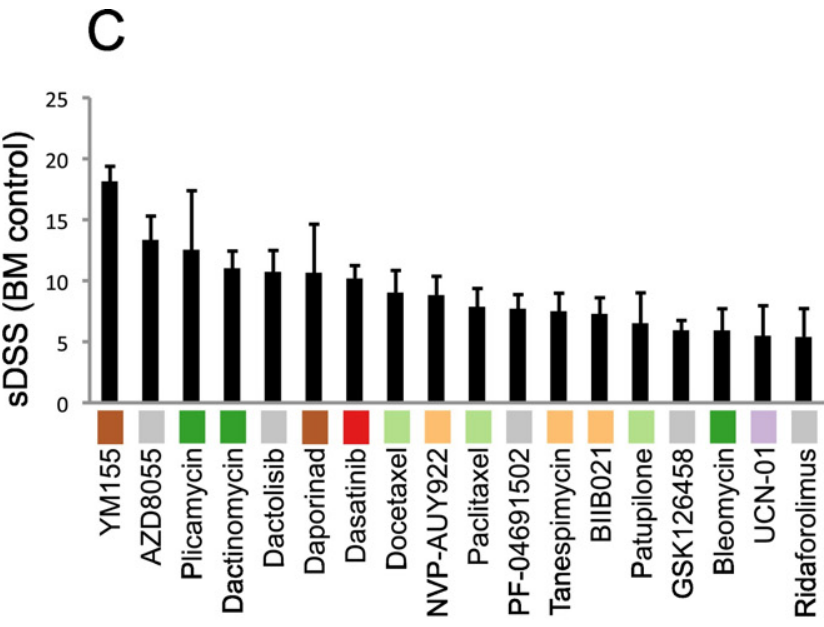
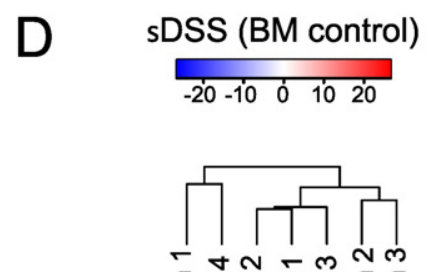

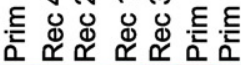

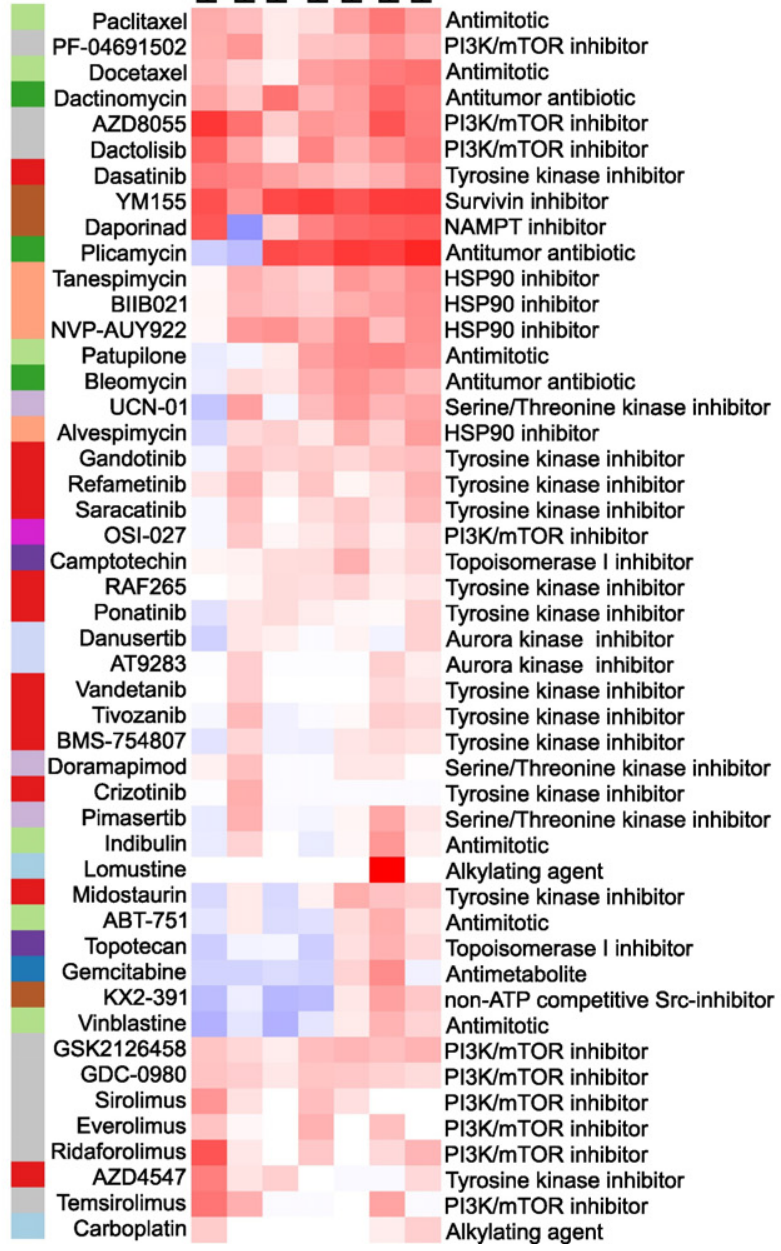


using the CellTiter-Glo luminescent assay (Promega Corporation, Madison, WI, USA) (see Supplementary methods for details).

\subsection{Scoring and clustering of drug response profiles}

To quantitatively profile the DSRT-wide drug responses of individual samples and to compare the drug responses between the AGCT samples, the model-based drug sensitivity score (DSS) was calculated [13]. DSS summarizes the area under the dose-response curve over the entire dose range.

The differential drug responses of the AGCT cells were scored by calculating the difference between the DSS of the patient sample and the average DSS of either the hGL or bone marrow cells (selective DSS, sDSS). The correlation analysis of drug screening data was performed between AGCT and KGN/COV434/hGL cells with and without applying a cutoff of 5 DSS units. Rank product analysis of differential drug response was performed between AGCT and KGN/COV434/hGL cells using the R-package "RankProd" at false discovery rate of $5 \%$ $($ FDR $<0.05)$ [21]. Clustering of the drug sensitivity profiles across the AGCT and control samples was performed using Ward's unsupervised hierarchical clustering procedure with Euclidean and Manhattan distance measures for the drug and sample profiles, respectively, using heatmap. 2 function in "gplots" $\mathrm{R}$ package. The associations between the DSS profiles were analyzed with Spearman's rank correlation coefficient using the R 3.1.0 statistical software platform (http://www.rproject.org/).

\subsection{Drug combination testing}

KGN and COV434 cell viability was tested in response to the drug combinations in a matrix format, with combinations of paclitaxel with either dasatinib, everolimus, AZD8055, or PF-04691502 (the dosages of each compound are provided in Supplementary Table S3). One thousand KGN or COV434 cells were plated per well on predrugged 384-well plates and incubated for $72 \mathrm{~h}$. The cell viability was measured with CellTiter-Glo. Drug combination synergy was quantified by comparing the observed joint inhibition level at each dose combination to the expected combination effect using the zero interaction potency model [22].

\subsection{RNA sequencing and variant analysis}

We performed RNA sequencing for five of the seven tested AGCT samples. Two samples were not sequenced due to tissue RNA quality. RNA-sequencing data on three unrelated hGL samples [23] were used for gene expression profiling and variant analysis. RNA was extracted, prepared and sequenced as described in the Supplementary methods. RNA-sequencing reads were corrected for adapters and trimmed for quality. Sequencing data were then mapped to human reference genome and assigned to genomic features. Transcript abundance was measured as Fragments Per Kilobase of exon per Million fragments mapped (FPKM) (for details, see Supplementary methods). Variant calling from RNA sequencing reads was performed on the AGCT samples using Genome Analysis Toolkit (GATK) [24]. For details see Supplementary methods.

\subsection{Human AGCT tissue microarray and immunohistochemistry}

A previously characterized AGCT tissue microarray [25] containing quadruple cores from 71 primary and 12 recurrent AGCTs was utilized for immunohistochemical staining of the drug targets. All histological
AGCT diagnoses were histologically re-evaluated (R.B.) and tested positive for FOXL2 (c.402C > G; C134W). As controls, normal ovarian tissue sections from two premenopausal women operated upon for cervical cancer were used.

The AGCT tissue microarray, tissue sections from all the available AGCT samples screened in this study (AGCT Prim 1, 2, and 3 and Rec 1 to 3 ), and control ovary sections were stained as previously described [25], with the following primary antibodies and dilutions: antisurvivin 1:400 (sc-10811, Santa Cruz Biotechnology Inc., CA, USA), anti-mTOR 1:50 (\#2983, Cell Signaling Technology Inc., MA, USA), anti-AKT (\#4691, Cell Signaling Technology Inc.) 1:2000, anti-c-Src (sc-8056, Santa Cruz Biotechnology Inc.) 1:800, anti-PDGFRA 1:1200 (sc-338, Santa Cruz Biotechnology Inc.), anti-PDGFRB 1:2000 (sc-339, Santa Cruz Biotechnology Inc.) and anti-EPHA5 1:1200 (sc-1014, Santa Cruz Biotechnology Inc.). Immunostaining of granulosa cells in small antral follicles was used as baseline (intermediate) staining to which the staining of tumor samples was compared. AGCT immunostaining was graded into high (including intermediate) or low (including negative) groups based on the staining intensity. Two researchers (N.A. and U.M.H.) performed the evaluation independently, and disagreements were resolved by a joint review.

\section{Results}

\subsection{AGCTs exhibited consistent drug response profiles}

A high-throughput DSRT platform [14] was used for screening the responses of three primary and four recurrent AGCTs, as well as those of GCT cell lines KGN and COV434 and hGL cells, to a panel of 230 compounds (Supplementary Table S1). The drug response profiles were quantified using the DSS [13] (Supplementary Table S2). Comparison of the average AGCT response profiles showed a high correlation with the KGN profile (Spearman's correlation $r=0.83$; Fig. 1A), whereas the correlation with the COV434 cell line and hGL reference was weaker ( $r=0.74$ and $r=0.68$, respectively, Fig. $1 \mathrm{~B}$ and $\mathrm{C})$. To exclude the effects of datapoints near the origin, we also repeated this comparison after filtering out insensitive drugs (DSS $<5$ ), which decreased the correlations (Supplementary Fig. S1). The functional similarities between the KGN cell line and AGCTs were further investigated by comparing their gene expression profiles, showing similarly high correlations, that also decreased when the non-expressed genes were filtered out (Supplementary Fig. S2). These results indicate that the KGN cell line mimics the AGCT response profile, thus providing a molecularlymatched cell model that is more readily exploitable than primary cultured AGCT cells.

\subsection{Selective DSS analysis revealed potential therapeutic compounds for AGCT}

AGCT drug selectivity was first evaluated against hGL cells. Among the AGCT samples, 14 compounds exhibited a high selective response (mean sDSS $\geq 5$ ) (Fig. 2A, Supplementary Fig. S3). Of the targeted compounds, the tyrosine kinase inhibitor dasatinib resulted in the highest selective sensitivity in all the tumors (mean sDSS $=11.3$ ). Three AGCTs exhibited markedly higher selectivity for dasatinib: Prim 1, Prim 3, and Rec 4 (sDSS of 15.0,13.3, and 13.7, respectively). The survivin inhibitor YM155, CHK1-inhibitor PF477736, and BCL-2/BCLXL-inhibitor obatoclax also showed relatively high selectivity (mean sDSS of $>5$ ) (Fig. 2A, B, Supplementary Fig. S3). However, the other tested BCL-2-inhibitors did not appear to have any selectivity for AGCT. Of the traditional chemotherapeutic antimitotic drugs, docetaxel and

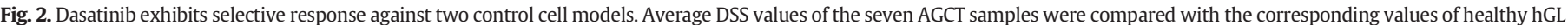

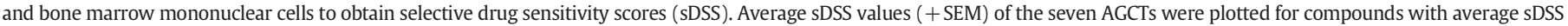

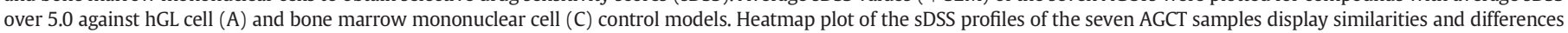
between the individual responses of the seven AGCT samples against the hGL cell (B) and the bone marrow mononuclear cell (D) controls. 
paclitaxel were the most effective (mean sDSS of 15.2 and 10.9, respectively). Six of the 14 selective compounds identified were antimitotic microtubule-targeting agents. It is likely that antimitotics and topoisomerase II inhibitors were overrepresented as selective compounds for AGCT due to the quiescent state of the hGL cells in vitro (Fig. 2B, Supplementary Fig. S3).

The AGCT response profile to bone marrow mononuclear (BM) cells was also compared to evaluate the drug sensitivity against healthy proliferating cells. Dasatinib and YM155 were both selective against the BM reference model (mean sDSS of 10.2 and 18.1, respectively, Fig. 2C). In total, 18 compounds exhibited high selectivity against the BM cells (mean sDSS, $\geq 5$ ), including several investigational compounds targeting mTOR/PI3K (five compounds) and heat shock protein 90 (three compounds) (Fig. 2D, Supplementary Fig. S4). Further, dasatinib and YM155 exhibited selective efficacy compared to a panel of 68 cancer cell lines derived from solid breast, ovarian, lung, and pancreatic cancers (Supplementary Fig. S5). Taken together, AGCTs showed selective sensitivity to dasatinib, PI3K/mTOR inhibitors, taxane antimitotics, and the survivin inhibitor YM155. Based on their clinical availability, the most interesting new selective compounds are dasatinib, the rapamycin ana$\log$ mTOR inhibitors everolimus and temsirolimus, and the taxanes paclitaxel and docetaxel.

Of interest, both the FOXL2 mutation-positive primary AGCT and KGN cells were sensitive to the tyrosine kinase inhibitor dasatinib, whereas the FOXL2 wild-type COV434 and hGL cells were resistant (Fig. 1). The response patterns to the other tyrosine kinase inhibitors did not differ between the FOXL2 mutation-positive and wild-type cells. Compared with primary AGCT cells, the GCT cell lines were more sensitive to conventional chemotherapeutics, such as antimitotic compounds, topoisomerase inhibitors, and tubulin stabilizers (Fig. 1). In addition, there were multiple compounds earlier implicated as potential AGCT treatment that had no effect on AGCT in our screen. Notably, hormone inhibitors had no effect on AGCT cell viability, similar to EGFR-inhibitors. Further, inhibition of RASRAF-MEK-ERK pathway showed only a minimal response in AGCT cells, whereas the GCT cell lines showed moderate sensitivity to these inhibitors (Supplementary Table S4).

\subsection{Dasatinib and mTOR/PI3K inhibitors synergistically inhibited AGCT cell viability in combination with paclitaxel}

Next, the efficacy of the selective targeted compounds dasatinib and mTOR inhibitors everolimus, AZD8055, and PF-04691502 were evaluated in dual combinations with the traditional chemotherapeutic paclitaxel in the two GCT cell lines KGN and COV434. The dual combination of dasatinib with paclitaxel revealed synergy across the dose range of 1-10 nM for dasatinib and 3-30 nM for paclitaxel in the KGN cells but not in the FOXL2 wild-type COV434 cells (Fig. $3 \mathrm{~A}$ and E, respectively). The highest synergy in KGN cells was reached with a 1-3 nM dose of dasatinib and $10 \mathrm{nM}$ dose of paclitaxel. The combinations of mTOR-inhibitors and paclitaxel also induced synergistic inhibition of KGN cell viability at relatively low concentrations. The highest synergy in KGN cells was reached at doses of between 30 and $100 \mathrm{nM}$ and 1-3 nM for everolimus and paclitaxel, respectively (Fig. 3B), whereas no synergy was detected in the COV434 cells at low drug concentrations (Fig. 3F). The ATP-competitive mTOR kinase inhibitors PF04691502 and AZD8055 also showed synergy with paclitaxel at a 1-10 nM dose of each compound (Fig. 3C and D, respectively), whereas the COV434 cells exhibited a negligent or low synergistic response (Fig. $3 \mathrm{G}$ and $\mathrm{H}$, respectively).
3.4. Selective drug sensitivity of AGCTs was conveyed by differential target expression rather than somatic mutations

Five of the seven tested AGCT samples (Prim 1 and Rec 1-4) were RNA-sequenced for gene expression analysis and mutation calling. Variant analysis of the RNA-sequencing data did not reveal known cancerassociated mutations in the samples tested. However, 108 potentially oncogenic gene variants were detected with COSMIC ID, including a frameshift insertion in ARF1 (ADP ribosylation factor-1) and stop-loss codon in HLA-G (Supplemental Fig. S6). ARF1 and HLA-G variants were present in four of five tumors (the mutation was absent in Rec 3 and Rec 2, respectively). Mutation calling for dasatinib and PI3K/mTOR inhibitor target genes was conducted using the RNA-sequencing data. Consistent with the lack of mutations in common cancer genes, neither codon-changing nor protein-truncating variants was found in dasatinib target genes or PI3K/mTOR pathway genes (Supplementary Fig. S6).

Dasatinib is a multitarget tyrosine kinase inhibitor targeting, for example, ABL1, Src family kinases, KIT, PDGFR, and ephrin receptors. Compared to hGL cells, the overall mRNA expression levels of several ephrin receptors (especially EPHA5 and EPHB3), PDGFRA, PDGFR, and KIT, were higher in the AGCTs (Fig. 4A). In addition, although most of the Src family members were abundantly expressed in AGCTs, only FYN expression was notably higher in the AGCTs than in the hGL cells. Corroborating the drug response profiles, genes encoding for the direct target proteins of the most efficient compounds were consistently expressed in the AGCTs at the mRNA level (Fig. 4A), and many members of the mTOR signaling pathway were also abundantly expressed in both AGCT and granulosa luteal cells (Fig. 4B).

Finally, the protein expression patterns of SRC, PDGFRA, PDGFRB, and EPHA5, as well as those of mTOR and its downstream signaling molecule AKT and survivin, were assessed in the tested AGCT samples (Prim $1-3$, Rec $1-3$ ) and in an AGCT tissue microarray (Supplementary Table S5, Supplementary Fig. S7). All these targets were abundantly expressed at the protein level. However, there were no significant differences between primary and recurrent tumors, and the expression levels were not correlated with patient survival (data not shown). Taken together, these data suggest that the observed drug sensitivity of AGCTs was conveyed by selective target expression rather than by known oncogenic mutations.

\section{Discussion}

In the present study, we systematically tested the drug sensitivity of primary cultured AGCT cells and GCT cell lines utilizing a highthroughput DSRT platform that comprised both traditional chemotherapeutics and targeted compounds (both investigational and approved). The results revealed novel targeted compounds that selectively decreased AGCT cell viability. Importantly, the study also revealed synergistic growth inhibition between the targeted compounds, dasatinib, and several mTOR-inhibitors, in combination with the current firstline treatment, paclitaxel.

The integration of data obtained from DSRT assays and molecular profiling has previously been shown to be a powerful strategy to identify individualized treatment options and potential biomarkers in hematological malignancies and prostate cancer [13-15]. This approach was applied herein to primary ovarian cancer cells derived from AGCT patients. To date, there are no established FOXL2 c. $402 C>G$ mutation-positive in vivo models to test for drug responses in AGCTs, further highlighting the importance of preclinical approaches to test new treatments for AGCT. DSRT is a promising

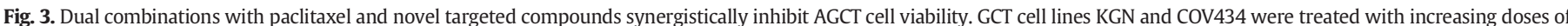

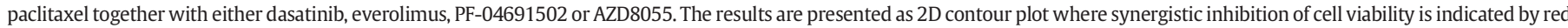

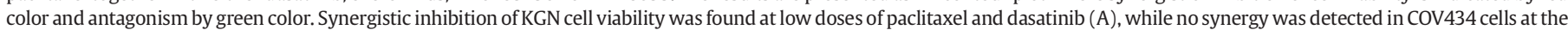
same doses (E). Combination treatment with paclitaxel and mTOR inhibitors also synergistically inhibited KGN cells (B-D) but not COV434 cell viability (F-H) at low doses. 


\section{Synergy score
$-30-20-10 \quad 0 \quad 10 \quad 20 \quad 30$}
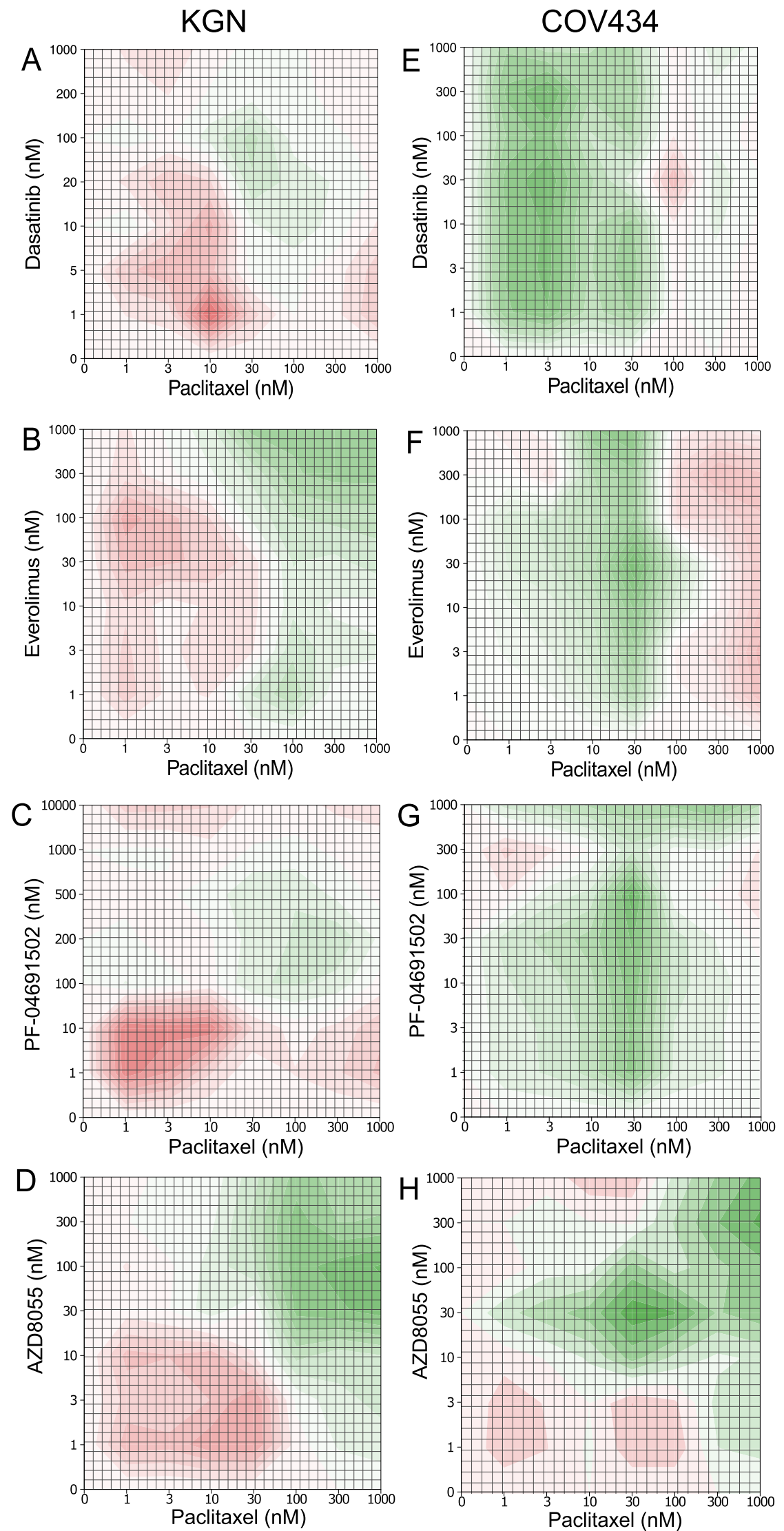

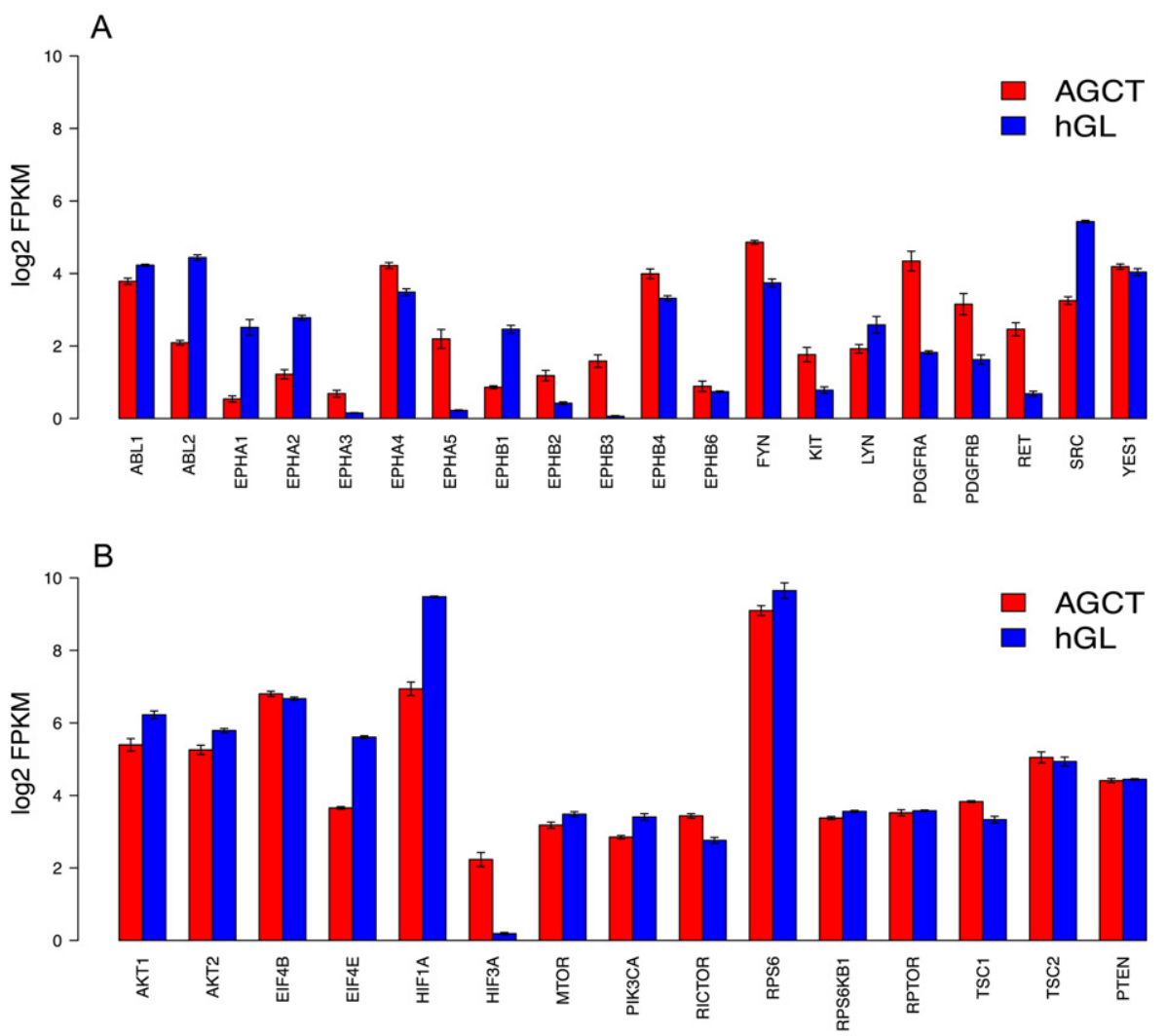

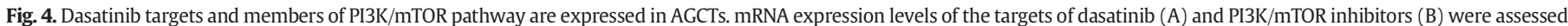

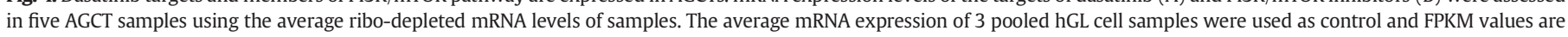
represented in log2 scale. Red bars implicate the AGCT samples and blue bars hGL samples. The bar plots present mean + SD.

functional approach to reveal novel treatment targets in this rare tumor subtype, which exhibits only little genetic variation between individual cases. Based on our study, responses of KGN cell line were mostly convergent with those seen in AGCT cells. Since also the gene expression levels were highly correlated between these cells, KGN can be regarded as a useful cell model for AGCT. RNA sequencing and subsequent mutation calling confirmed the lack of mutations in known oncogenes in the AGCT patients, indicating that drug sensitivity was mainly conveyed by selective target expression. Even though our sample size was limited, we think these results are representative for AGCT, considering the similarity of transcriptomic profiles among the individual tumors. Of the detected variants ARF1 is a GTP-binding molecule, known to have an essential role in phosphorylation of SRC [26]whereas HLA-G plays a role in tumor immune surveillance [27]. As the variant types for ARF1 and HLA-G were frameshift insertion and stoploss type respectively, they are expected to affect the protein function. However the clinical significance of these variants detected herein in a subset of AGCTs will require further study.

Dasatinib is a tyrosine kinase inhibitor that is widely used in the treatment of chronic myeloid leukemia [28], showing efficacy also against solid tumors when used in combination with other drugs $[29,30]$. Although dasatinib primarily targets SRC and ABL, it also inhibits a variety of other tyrosine kinases, such as PDGFR and ephrin receptors [31]. Consistent with an earlier report of the effects of an SRC inhibitor in KGN cells [32], the present study showed that dasatinib strongly and selectively inhibited AGCT cell viability. Interestingly, dasatinib was effective in FOXL2-mutation positive AGCTs and the KGN cell line, whereas the responses in FOXL2 wild-type COV434 cells and hGL reference cells were significantly weaker. Moreover, the synergistic effects were selective to AGCTs, as the synergy between dasatinib and paclitaxel was evident in KGN cells, whereas no synergy was seen in the FOXL2 wild-type COV434 cells. However, this possible association between the dasatinib response and FOXL2 mutation will need to be further validated in functional studies. Given that the other KIT or PDGFR inhibitors in the studied drug collection did not show a selective response, the cell viability inhibiting effect of dasatinib is likely to be conveyed through specific ephrin receptors or Src family kinases. Consistently, most of the dasatinib targets were highly expressed at the mRNA level and also showed strong expression in the majority of AGCTs at the protein level. The present study found no mutations in the dasatinib target genes, and further studies are required to confirm the role of both ephrin receptors and Src family kinases in AGCTs.

The PI3K/Akt/mTOR signaling pathway regulates cell growth and proliferation, and activation of MTOR and Akt has been shown to promote granulosa cell proliferation and differentiation [33]. This signaling pathway is also active in AGCT [34], and treatment with the mTOR inhibitor everolimus has been shown to reduce tumor growth in a mouse GCT model [35]. mTOR inhibitors selectively inhibited AGCT cell viability in our systematic screen, and many key components of the mTOR pathway were abundantly expressed in AGCTs at the mRNA level. In addition, highly expressed survivin has been shown to be regulated by the mTOR pathway [36]. Survivin is also one of the downstream effectors of the beta-catenin pathway, which promotes malignant tumorigenesis in granulosa cells [37]. Although the survivin inhibitor YM155 has shown promising results in preclinical models, the clinical efficacy of YM155 is limited, and its drug development has recently been discontinued. However, in a recent study with ovarian cancer patients, survivin was used as an antigen to generate cancer-specific vaccine-induced immune responses [38]. Altogether, these findings implicate a potential role for mTOR inhibitors in the treatment of AGCTs and support the potential of survivin as a therapeutic target. 
In accordance with clinical experience, paclitaxel and docetaxel were the most efficient traditional chemotherapeutics for AGCTs. Importantly, the present study revealed significant synergy between paclitaxel and dasatinib and between paclitaxel and mTOR inhibitors. In epithelial ovarian cancer cells, dasatinib has been shown to enhance the antitumor activities of paclitaxel and carboplatin, and this combination has also been tested in a phase I trial, with evidence of clinical activity $[30,39]$. Synergy between mTOR inhibitors and paclitaxel has also been reported in breast cancer cells [40]. The lack of chromosomal instability and low intra- and intertumoral heterogeneity in AGCTs may render these cancers sensitive to such synergistic treatments in the clinical setting.

In conclusion, the present study introduced a systematic drug screening approach in ovarian cancer cells to reveal novel potential compounds and drug combinations, which can be further tested in clinical trials. Molecular profiling revealed a relatively homogenous set of AGCT tumors, in which the drug sensitivities were conveyed by their target gene expressions. The selective response of dasatinib in all tested AGCTs highlights dasatinib as the most potential drug for AGCTs in our screen. The combination of dasatinib and paclitaxel has been shown to be well tolerated in clinical trials of other tumor types [30]. Importantly, the herein observed synergy of dasatinib and paclitaxel urges clinical testing of this combination in relapsed or aggressive AGCTs.

Supplementary data to this article can be found online at http://dx. doi.org/10.1016/j.ygyno.2016.12.016.

\section{Disclosure of potential conflicts of interest}

No conflicts of interest were disclosed.

\section{Funding}

This study was supported by grants from the Academy of Finland (grants 269 862, 272 437, 272 577, 277 293, 279 163, 295504 and 292 611), Biocenter Finland, Cancer Society of Finland, FIMM National Network in Molecular Medicine, Doctoral Programme in Biomedicine (DPBM), Helsinki University Hospital Research Funds, Jane and Aatos Erkko Foundation, Paulo Foundation, Sigrid Jusélius Foundation, and Sladjana M. Crosley Foundation for GCT Research.

\section{Acknowledgements}

We thank Dr. Timo Tuuri for providing the hGL cells for DSRT. Prof. Reiner Veitia, Prof. Andres Salumets and Dr. Agne Velthut-Meikas are thanked for providing the mural granulosa cell RNA sequencing data. We thank Dr. Astrid Murumägi, Dr. Päivi Östling, Dr. Akira Hirasawa and Mr. Prson Gautam for their help with the drug testing reference panel. Dr. Petteri Hintsanen, Mr. Prson Gautam, Dr. Pirkko Mattila and Mr. Jesus M. Lopez Marti are acknowledged for their contributions to the RNA sequencing. We thank all the patients for donating samples for research, and all the personnel at the Department of Obstetrics and Gynecology for supporting the patient recruitment.

\section{References}

[1] S. Jamieson, P.J. Fuller, Molecular pathogenesis of granulosa cell tumors of the ovary, Endocr. Rev. 33 (1) (2012 Feb) 109-144

[2] S.P. Shah, M. Kobel, J. Senz, R.D. Morin, B.A. Clarke, K.C. Wiegand, et al., Mutation of FOXL2 in granulosa-cell tumors of the ovary, N. Engl. J. Med. 360 (26) (2009 Jun 25) 2719-2729.

[3] S. Bryk, A. Farkkila, R. Butzow, A. Leminen, M. Heikinheimo, M. Anttonen, et al., Clinical characteristics and survival of patients with an adult-type ovarian granulosa cell tumor: a 56-year single-center experience, Int. J. Gynecol. Cancer 25 (1) (2015 Jan) 33-41.

[4] G. Mangili, J. Ottolina, A. Gadducci, G. Giorda, E. Breda, A. Savarese, et al., Long-term follow-up is crucial after treatment for granulosa cell tumours of the ovary, Br. J. Cancer 109 (1) (2013 Jul 9) 29-34.

[5] A. Farkkila, N. Andersson, R. Butzow, A. Leminen, M. Heikinheimo, M. Anttonen, et al., HER2 and GATA4 are new prognostic factors for early-stage ovarian granulosa cell tumor-a long-term follow-up study, Cancer Med. 3 (3) (2014 Jun) 526-536.
[6] A.T. Evans 3rd, T.A. Gaffey, G.D. Malkasian Jr., J.F. Annegers, Clinicopathologic review of 118 granulosa and 82 theca cell tumors, Obstet. Gynecol. 55 (2) (1980 Feb) 231-238.

[7] H.D. Homesley, B.N. Bundy, J.A. Hurteau, L.M. Roth, Bleomycin, etoposide, and cisplatin combination therapy of ovarian granulosa cell tumors and other stromal malignancies: a Gynecologic Oncology Group study, Gynecol. Oncol. 72 (2) (1999 Feb) 131-137.

[8] J. Brown, H.S. Shvartsman, M.T. Deavers, L.M. Ramondetta, T.W. Burke, M.F. Munsell, et al., The activity of taxanes compared with bleomycin, etoposide, and cisplatin in the treatment of sex cord-stromal ovarian tumors, Gynecol. Oncol. 97 (2) (2005 May) 489-496.

[9] H.S. van Meurs, M.R. Buist, A.M. Westermann, G.S. Sonke, G.G. Kenter, J. van der Velden, Effectiveness of chemotherapy in measurable granulosa cell tumors: a retrospective study and review of literature, Int. J. Gynecol. Cancer 24 (3) (2014 Mar) 496-505.

[10] E.A. Eisenhauer, P. Therasse, J. Bogaerts, L.H. Schwartz, D. Sargent, R. Ford, et al., New response evaluation criteria in solid tumours: revised RECIST guideline (version 1.1), Eur. J. Cancer 45 (2) (2009 Jan) 228-247.

[11] B.A. Benayoun, M. Anttonen, D. L'Hote, M. Bailly-Bechet, N. Andersson, M. Heikinheimo, et al., Adult ovarian granulosa cell tumor transcriptomics: prevalence of FOXL2 target genes misregulation gives insights into the pathogenic mechanism of the p.Cys134Trp somatic mutation, Oncogene 32 (22) (2013 May 30) 2739-2746.

[12] J.H. Kim, S. Yoon, M. Park, H.O. Park, J.J. Ko, K. Lee, et al., Differential apoptotic activities of wild-type FOXL2 and the adult-type granulosa cell tumor-associated mutant FOXL2 (C134W), Oncogene 30 (14) (2011 Apr 7) 1653-1663.

[13] B. Yadav, T. Pemovska, A. Szwajda, E. Kulesskiy, M. Kontro, R. Karjalainen, et al., Quantitative scoring of differential drug sensitivity for individually optimized anticancer therapies, Sci. Rep. 4 (2014 Jun 5) 5193.

[14] T. Pemovska, M. Kontro, B. Yadav, H. Edgren, S. Eldfors, A. Szwajda, et al., Individualized systems medicine strategy to tailor treatments for patients with chemorefractory acute myeloid leukemia, Cancer Discov. 3 (12) (2013 Dec) 1416-1429.

[15] K. Saeed, V. Rahkama, S. Eldfors, D. Bychkov, JP. Mpindi, B. Yadav, et al., Comprehensive drug testing of patient-derived conditionally reprogrammed cells from castration-resistant prostate cancer, Eur. Urol. (2016 May 5).

[16] A. Kyronlahti, M. Kauppinen, E. Lind, L. Unkila-Kallio, R. Butzow, J. Klefstrom, et al., GATA4 protects granulosa cell tumors from TRAIL-induced apoptosis, Endocr. Relat. Cancer 17 (3) (2010 Jul 28) 709-717.

[17] Y. Nishi, T. Yanase, Y. Mu, K. Oba, I. Ichino, M. Saito, et al., Establishment and characterization of a steroidogenic human granulosa-like tumor cell line, KGN, that expresses functional follicle-stimulating hormone receptor, Endocrinology 142 (1) (2001 Jan) 437-445.

[18] C.A. van den Berg-Bakker, A. Hagemeijer, E.M. Franken-Postma, V.T. Smit, P.J. Kuppen, H.H. van Ravenswaay Claasen, et al., Establishment and characterization of 7 ovarian carcinoma cell lines and one granulosa tumor cell line: growth features and cytogenetics, Int. J. Cancer 53 (4) (1993 Feb 20) 613-620.

[19] F.T. Shi, A.P. Cheung P.C. Leung, Growth differentiation factor 9 enhances activin ainduced inhibin B production in human granulosa cells, Endocrinology 150 (8) (2009 Aug) 3540-3546.

[20] P. Gautam, L. Karhinen, A. Szwajda, S.K. Jha, B. Yadav, T. Aittokallio, et al., Identification of selective cytotoxic and synthetic lethal drug responses in triple negative breast cancer cells, Mol. Cancer 15 (1) (2016 May 10) (34-016-0517-3).

[21] F. Hong, B. Wittner, R. Breitling, C. Smith, F. Battke, RankProd: Rank Product Method for Identifying Differentially Expressed Genes With Application in Meta-analysis, R Package Version, 2(0), 2011.

[22] B. Yadav, K. Wennerberg, T. Aittokallio, J. Tang, Searching for Drug Synergy in Complex Dose-Response Landscapes Using an Interaction Potency Model, 2015.

[23] A. Velthut-Meikas, J. Simm, T. Tuuri, J.S. Tapanainen, M. Metsis, A. Salumets, Research resource: small RNA-seq of human granulosa cells reveals miRNAs in FSHR and aromatase genes, Mol. Endocrinol. 27 (7) (2013 Jul) 1128-1141.

[24] A. McKenna, M. Hanna, E. Banks, A. Sivachenko, K. Cibulskis, A. Kernytsky, et al., The Genome Analysis Toolkit: a MapReduce framework for analyzing next-generation DNA sequencing data, Genome Res. 20 (9) (2010 Sep) 1297-1303.

[25] M. Anttonen, L. Unkila-Kallio, A. Leminen, R. Butzow, M. Heikinheimo, High GATA-4 expression associates with aggressive behavior, whereas low anti-Mullerian hormone expression associates with growth potential of ovarian granulosa cell tumors, J. Clin. Endocrinol. Metab. 90 (12) (2005 Dec) 6529-6535.

[26] S. Schlienger, R.A. Ramirez, A. Claing, ARF1 regulates adhesion of MDA-MB-231 invasive breast cancer cells through formation of focal adhesions, Cell. Signal. 27 (3) (2015 Mar) 403-415.

[27] A. Lin, W.H. Yan, HLA-G expression in cancers: roles in immune evasion, metastasis and target for therapy, Mol. Med. 24 (2015 Aug).

[28] A. Hochhaus, H. Kantarjian, The development of dasatinib as a treatment for chronic myeloid leukemia (CML): from initial studies to application in newly diagnosed patients, J. Cancer Res. Clin. Oncol. 139 (12) (2013 Dec) 1971-1984.

[29] J.C. Araujo, P. Mathew, A.J. Armstrong, E.L. Braud, E. Posadas, M. Lonberg, et al., Dasatinib combined with docetaxel for castration-resistant prostate cancer: results from a phase 1-2 study, Cancer 118 (1) (2012 Jan 1) 63-71.

[30] A.A. Secord, D.K. Teoh, W.T. Barry, M. Yu, G. Broadwater, L.J. Havrilesky, et al., A phase I trial of dasatinib, an SRC-family kinase inhibitor, in combination with paclitaxel and carboplatin in patients with advanced or recurrent ovarian cancer, Clin. Cancer Res. 18 (19) (2012 Oct 1) 5489-5498.

[31] J.C. Montero, S. Seoane, A. Ocana, A. Pandiella, Inhibition of SRC family kinases and receptor tyrosine kinases by dasatinib: possible combinations in solid tumors, Clin. Cancer Res. 17 (17) (2011 Sep 1) 5546-5552.

[32] S. Jamieson, P.J. Fuller, Tyrosine kinase inhibitors as potential therapeutic agents in the treatment of granulosa cell tumors of the ovary, Int. J. Gynecol. Cancer 25 (7) (2015 Sep) 1224-1231.

[33] Y. Cheng, J. Kim, X.X. Li, A.J. Hsueh, Promotion of ovarian follicle growth following mTOR activation: synergistic effects of AKT stimulators, PLoS One 10 (2) (2015 Feb 24), e0117769. 
[34] M. Miyazawa, M. Yasuda, M. Fujita, K. Hirabayashi, T. Hirasawa, H. Kajiwara, et al. Granulosa cell tumor with activated mTOR-HIF-1alpha-VEGF pathway, J. Obstet. Gynaecol. Res. 36 (2) (2010 Apr) 448-453.

[35] C. Rico, M.N. Lague, P. Lefevre, M. Tsoi, A. Dodelet-Devillers, V. Kumar, et al., Pharmacological targeting of mammalian target of rapamycin inhibits ovarian granulosa cell tumor growth, Carcinogenesis 33 (11) (2012 Nov) 2283-2292.

[36] V. Vaira, C.W. Lee, H.L. Goel, S. Bosari, L.R. Languino, D.C. Altieri, Regulation of survivin expression by IGF-1/mTOR signaling, Oncogene 26 (19) (2007 Apr 26) 2678-2684.

[37] D. Boerboom, M. Paquet, M. Hsieh, J. Liu, S.P. Jamin, R.R. Behringer, et al. Misregulated Wnt/beta-catenin signaling leads to ovarian granulosa cell tumor development, Cancer Res. 65 (20) (2005 Oct 15) 9206-9215.
[38] N.L. Berinstein, M. Karkada, A.M. Oza, K. Odunsi, J.A. Villella, J.J. Nemunaitis, et al. Survivin-targeted immunotherapy drives robust polyfunctional T cell generation and differentiation in advanced ovarian cancer patients, Oncoimmunology 4 (8) (2015 May 7), e1026529.

[39] J. Xiao, M. Xu, T. Hou, Y. Huang, C. Yang, J. Li, Dasatinib enhances antitumor activity of paclitaxel in ovarian cancer through Src signaling, Mol. Med. Rep. 12 (3) (2015 Sep) 3249-3256

[40] W.H. Mondesire, W. Jian, H. Zhang, J. Ensor, M.C. Hung, G.B. Mills, et al., Targeting mammalian target of rapamycin synergistically enhances chemotherapy-induced cytotoxicity in breast cancer cells, Clin. Cancer Res. 10 (20) (2004 Oct 15) 7031-7042. 